males and 1.37 (95\%-IC: 1.26-1.48) in females, while radon related RRs were not statically significant. Adjusting for socio-demographic factors, the RRs by occupation decreased by $16 \%$ and $4 \%$ on average in men and women, and by $4 \%$ and $6 \%$ when adjusting for smoking, but remained statistically significant.

Conclusions The results suggest the presence of occupational exposures to lung carcinogens in addition to non-occupational factors. Longer follow-up and analysis by histological types of lung cancer are needed to improve the estimates of occupational lung cancer.

\section{0-303 THE ROLE OF OCCUPATIONAL EXPOSURES IN LUNG CANCER RISK AMONG WOMEN: PRELIMINARY RESULTS FROM A POOLED CASE-CONTROL STUDY OF LUNG CANCER}

${ }^{1}$ Mengting Xu, Vikki Ho, Jack Siemiatycki. 'University of Montreal, Canada

10.1136/OEM-2021-EPI.76

Introduction Lung cancer is the most common cause of cancer death among women. However, little is known regarding occupational risk factors for lung cancer in women.

Objective To investigate possible associations between selected occupational agents and lung cancer risk among women.

Methods We pooled data from ten case-control studies of lung cancer with detailed lifetime occupational and smoking history. The current analysis was restricted to working women, including 3040 cases and 4186 controls. To assess occupational exposure, we used the Canadian Job-Exposure Matrix (CANJEM). Linking participants' jobs to the CANJEM allows the estimation of probability, and frequency of exposure to a list of 258 agents. This analysis was restricted to 36 most prevalent occupational agents in our sample of women. The association between lung cancer risk and lifetime ever exposure, duration of exposure, and cumulative exposure for each agent was estimated in separate logistic regression models, adjusted for smoking and other selected covariates.

Results Most agents we examined were not associated with lung cancer. We observed an increased risk of lung cancer among women occupationally exposed to cooking fumes for over 10 years $(\mathrm{OR}(95 \% \mathrm{CI})=1.73(1.09-2.82))$. Statistically significant decreased risks of lung cancer were observed among women exposed to various textile fibres, especially among long-duration workers. The results regarding the various textile agents have not been mutually controlled yet. When restricting to never smokers, increased risks of lung cancer were observed among women exposed to metallic dust, isopropanol, and aliphatic alcohols, with OR point estimates for ever exposure ranging from 1.5 to 1.7 .

Conclusion Our preliminary results indicate that occupational exposure to cooking fumes is associated with an increased lung cancer risk in women, while exposures to various textile fibres seem to be associated with a decreased lung cancer risk.

\section{0-321 EXPLORATION OF OCCUPATIONS AS RISK FACTORS FOR LUNG CANCER IN MULTIPLE EXPOSURE HIERARCHICAL AND PENALIZATION MODELS}

${ }^{1}$ Calvin Ge, Susan Peters, Ann Olsson, Joachim Schuz, Hans Kromhout, Kurt Straif, Roel Vermeulen, Thomas Brüning, Lützen Portengen. ' Utrecht University, Netherlands

10.1136/OEM-2021-EPI.77
Introduction We used hierarchical and penalization models to explore occupational risks associated with lung cancer while accounting for exposures to multiple known carcinogenic exposures.

Methods We pooled lung cancer case-control study subjects from 14 European and Canadian studies. Associations between employment in 1,506 five-digit ISCO-68 occupations and lung cancer were screened using Bayesian hierarchical and lasso penalized regressions accounting for age, smoking, sex, study, and fully quantitative exposures to six known occupational lung carcinogens: asbestos, chromium, diesel engine exhaust, nickel, PAHs, and silica. False positive findings in the penalization model were controlled using stability selection with specified family-wise error rates. Lung cancer odds ratios for selected occupations were calculated using unconditional logistic regression model with identical covariates.

Results Our study included 16,901 cases and 20,965 controls. Jobs selected by the hierarchical and penalization models were similar. Occupations with positive associations with lung cancer after controlling for the known carcinogens included building painters (OR: 1.40; $95 \mathrm{CI}: 1.17,1.67$ ), carpenters (OR: 1.77; 95 CI: 1.36, 2.33), and paviours (OR: 3.91; 95 CI: $1.75,9.61)$.

Conclusion We demonstrated viable agnostic approaches in identifying employment risk factors for lung cancer. Future work involves investigations of factors that contribute to the observed elevated cancer risks.

\section{0-335 OCCUPATIONAL PHYSICAL ACTIVITY AND LUNG CANCER RISK AMONG FOUR COHORTS OF THE CANADIAN PARTNERSHIP FOR TOMORROW'S HEALTH PROJECT}

${ }^{1}$ Romain Pasquet, Yun Zhu, Emmanuelle Batisse, Jérôme Lavoué, Marie-Elise Parent, Jack Siemiatycki, Anita Koushik, Vikki Ho. ${ }^{1}$ Centre de recherche du Centre hospitalier de I'Université de Montréal (CRCHUM), Canada

\subsection{6/OEM-2021-EPI.78}

Introduction Recreational physical activity (PA) has been associated with reduced lung cancer risk. Occupational-related PA may be one of the most important sources of PA, but the few studies that have examined the occupational PA-lung cancer relationship have produced inconsistent result.

Objective To investigate the association between occupational $\mathrm{PA}$ and lung cancer risk.

Methods A case-cohort study was nested among four cohorts of the Canadian Partnership for Tomorrow's Health Project. Through linkage of each cohort to their respective provincial cancer registry, 453 incident lung cancer cases diagnosed between 2009 and 2016 were identified; a random sub-cohort of 2,435 participants was sampled at baseline. Data on the longest-held job and risk factors were gathered via a harmonized questionnaire. Using a database generated by our team on the energy expenditures associated with almost 3,600 job titles, metabolic equivalent of tasks (METs) were assigned to the longest-held job of each participant. In total, 335 cases (139 men; 196 women) and 2,320 sub-cohort members (879 men; 1,441 women) with assignable job codes and MET values were included in this analysis. Multivariable logistic regression models, with normal or robust variance estimators, were used to estimate study-specific odds ratios (ORs) and 95\% confidence intervals (CIs) for the relationship between occupational PA (categorized in tertiles) and lung cancer risk, while 
adjusting for smoking and lung cancer risk factors identified using directed acyclic graphs. The ORs were then pooled using a random-effects model.

Results Compared with low occupational PA as reference, subjects with medium and high occupational PA had ORs of 0.64 (95\% CI: $0.38-1.07)$ and 0.73 (95\% CI: 0.39-1.34), respectively. The ORs were similar by sex and by smoking status (never vs. ever smokers).

Conclusion Our findings suggest that, like recreational PA, occupational PA may protect against lung cancer risk.

\section{0-415 CHANGING TRENDS FOR MESOTHELIOMA IN CANADA AND THEIR IMPLICATIONS}

${ }^{1}$ Paul Demers, Hunter Warden, Lillian Tamburic, Catherine Slavik, Chris McLeod. 'Ontario Health, Canada

\subsection{6/OEM-2021-EPI.79}

Introduction Canada was once the world's largest producer of asbestos, but exposure has been decreasing since the 1970's due to restrictions on use, lower occupational exposure limits, closing of mines, and a ban in 2018 .

Objectives The objectives of this study were to evaluate how rates of mesothelioma in Ontario and British Columbia (BC), which together constitute over 50\% of the Canadian population, have changed over time, by sex, age, geographical region and tumour site.

Methods The Ontario and BC Cancer Registries were used to identify 4,146 and 1,659 malignant mesothelioma cases between the years 1993-2017 and 1992-2016, respectively. Time trends were examined by sex, age, and anatomical site. Birth cohort models for Ontario were fit using US National Cancer Institute's age-period-cohort analysis web tool.

Results Ontario incidence rates for mesothelioma climbed from $1.0 / 100,000$ in 1993 until 2012 when rates plateaued at approximately 1.6. In BC the rate climbed from 1.1 in 1993 to $1.7 / 100,000$ in 2003 , when it began to plateau. Although female rates are much lower than male, they continue to steadily rise in both provinces. Rates among people over the age of 70 rose dramatically over time, while rates were steady or dropped among people below the age of 50 in both provinces. Peritoneal rates continue to rise in Ontario, but not BC. Relative to the 1921-25 birth cohort, male incidence rate ratios increased until peaking in 1936-40. Rate ratios for subsequent male cohorts decreased. In contrast, using the same reference period, the risk in women rose slowly with successive birth cohorts, though confidence limits were wide due to the low case counts.

Conclusion These complex changes over time may be due to major reductions in exposure in the 1970's, longer latency periods associated with lower levels of exposure, and the growing importance of environmental exposures.

\section{Methods}

\section{0-15 OCCUPATIONAL HEALTH: A MULTI-COHORT JOB TITLE CLEANING PROJECT BY ALGORITHM}

'Ellen Sweeney, Christopher Baker, Mohammad Sadnan Al Manir, Deobrah Addey, Yunsong Cui, Hicks Jason, Cheryl Peters, Grace Shen Tu, Jennifer Vena, Anil Adisesh. ${ }^{1}$ Dalhousie University, Canada

10.1136/OEM-2021-EPI.80
Introduction Occupational data in prospective cohort studies is often underutilized due to the human and financial resources required to code open-ended text, such as job titles. Recognizing the value of occupational data in health research, as well as potential errors associated with manual coding, an Automated Coding Algorithm (ACA)-NOC algorithm was developed utilizing a Natural Language Processing approach.

Objectives We tested the ACA-NOC algorithm on two regional cohorts of a pan-Canadian cohort study, which represents the largest dataset an algorithm of this kind has been applied to. This process will harmonize and greatly expand the utility of the occupational data, enrich the research platforms, and further refine the efficiency of the algorithm.

Methods The ACA-NOC algorithm was tested on data from the Canadian Partnership for Tomorrow's Health (CanPath), a longitudinal cohort examining the role of genetic, environmental, lifestyle, and behavioural factors in the development of cancer and chronic disease. Using an iterative and interactive approach, the algorithm was applied to job title data from 111,000 questionnaires from two regional cohorts, coding the data to the Canadian National Occupation Classification (NOC) system. The algorithm was further refined based on each round of analysis, increasing the quantity of accurately coded data.

Results Results from this research demonstrate the ability to refine the ACA-NOC algorithm with a $10 \%$ overall improvement in exact matching from the baseline algorithm. There were also instances where the algorithm performance was superior to the manual coding. The utilization of the algorithm offers significant savings in time, human resources and cost compared to a singular manual coding approach.

Conclusions The coding and harmonization of this multicohort data demonstrates the value of the ACA-NOC algorithm, while increasing the utility of the CanPath data and research related to occupational health. Future research may involve comparisons between CanPath and international cohorts.

\section{0-299 AN APPLICATION OF A PSEUDOLIKELIHOOD APPROACH IN A COUNTER-MATCHED STUDY OF BLADDER CANCER IN A COHORT OF STEEL WORKERS EXPOSED TO METALWORKING FLUID MISTS}

${ }^{1}$ Michel Grzebyk, Mohamed Arnouss, Regis Colin, Eve Bourgkard, Pascal Wild. 'French National Research and Safety Institute, France

\subsection{6/OEM-2021-EPI.81}

Objectives To compare partial likelihood and pseudolikelihood approaches in a nested case-control study under countermatching design in the presence of time varying-covariates.

Methods Within a prospective cohort of 17603 French steel workers, cases were newly diagnosed with a histologically-confirmed carcinoma of the bladder in 2006-2012. Three controls per case matched on age at diagnosis were randomly selected following a countermatched sampling scheme using a four-strata surrogate time-varying exposure to metalworking fluid (MWF) mists covariate, assessed by a job-exposure matrix. Cases $(n=84)$ and controls $(n=251)$ provided information during questionnaire interviews concerning smoking history and occupational exposures history that were compiled by experts to assess occupational exposure to straight MWFs, 